\title{
Bone metastases from osteosarcoma
}

\author{
M. San-Julian; P. Diaz-de-Rada; E. Noain; L. Sierrasesumaga
}

M. San-Julian; P. Diaz-de-Rada; E. Noain
Department of Orthopaedics, University Clinic.
University of Navarra, 31080-Pamplona, Spain
e-mail: Msjulian@unav.es
Tel.: +34-948-255400, Fax: +34-948-296500
L. Sierrasesumaga
Department of Pediatrics, University of Navarra
Pamplona, Spain

\begin{abstract}
Bony metastases in patients with osteosarcoma are unusual and normally appear late in the course of the disease. We report our experience with eight such patients, four with solitary and four with multiple metastases. Those with solitary metastases were treated as new tumours with neoadjuvant chemotherapy and surgery. Three remain alive with no evidence of disease at 5, 7 and 8 years follow-up respectively. Histology and response to neoadjuvant chemotherapy was similar in both the primary and metastatic lesions and is a predictive factor of outcome. Those with multiple metastases were treated by palliative measures, and none survived. We conclude that resection of solitary metastases from osteosarcoma after neoadjuvant chemotherapy can be curative.
\end{abstract}

\section{RÉSUMÉ}

Les métastases osseuses chez les patients atteints d'ostéosarcome sont rares et n'apparaissent que tardivement dans l'évolution de la maladie. Nous rapportons notre expérience avec 8 patients, 4 ayant uniquement des métastases osseuses et 4 des métastases multiples. Ceux qui présentaient une seule métastase ont été traité comme une tumeur primitive avec chimiothérapie néoadjuvante et chirurgie. Trois d'entre eux sont encore vivants sans évidence de maladie après 5,7 et 8 ans de suivi. L'histologie et la réponse à la chimiothérapie néoadjuvante ont été similaires tant pour la lésion primaire que pour les métastases. Les patients avec métastases multiples ont été traité avec des mesures palliatives. Aucun d'eux n'a survécu. On peut conclure que la résection de ces métastases après chimiothérapie néoadjuvante peut être utilisée avec une intention curative en cas de métastase osseuse unique. 


\section{INTRODUCTION}

The dissemination of metastases from sarcoma of bone is usually haematogenous, and the most frequent site for metastases is the lung [1, 2]. Treatment of lung metastases by surgery may improve survival rates in these patients [5, 6, 8, 9]. Skeletal metastases are less common and usually develop later in the disease. The aim of this paper was to analyse treatment and its outcome for patients with bony metastases from osteosarcoma.

\section{PATIENTS AND METHODS}

We treated 183 patients with osteosarcoma at the University Clinic of Navarra between 1979 and 2001, 136 of whom received the complete protocol of treatment, trying to save the limb in all cases $[13,14,16]$. Primary surgical procedure was conservative in all but one case, regardless of their Enneking staging. Patients with more than one bony lesion at the time of diagnosis (three cases) could be considered as having synchronous multifocal osteosarcoma rather than bony metastases and therefore were not included in this study. Patients with skip metastases at the time of diagnosis were also excluded.

Eight patients presented with bony metastases during routine follow-up. Mean patient age was 15 (11-30) years. Primary tumour site was the femur (four cases), tibia (three cases) and humerus (one case). Histological subtype was osteoblastic (five cases), chondroblastic (two cases) and telangiectatic (one case). Bony metastases appeared at a mean of 30 (19-68) months after diagnosis of the primary tumour. Four were solitary bony metastases and four were multiple, or combined with lung metastases. Solitary metastases sites were distal femur (two cases), acetabulum (one case) and lumbar spine (one case). In all cases, the histology was the same as that of the primary lesion.

\section{RESULTS}

When there were multiple bony metastases, (four cases), palliative treatment was undertaken with chemotherapy. Unusual drugs were used in an attempt to control the disease, including radioactive strontium, topotecan and melphalan. Palliative surgery was undertaken for pain control in two cases, one involving the facial nerve and one the sciatic nerve. All patients died at a mean of 16 months after detection of the bony metastases.

When solitary metastases were detected, a biopsy was performed and the treatment for the metastases was similar as that for the primary lesion, including neoadjuvant chemotherapy and surgery. The nature of the surgical procedure depended on the site of the lesion.

1. A wide resection and reconstruction with composite allograft-prosthesis was undertaken for a metastasis in the knee.

2. A marginal resection and reconstruction with intercalary allograft was undertaken for a metastasis in the distal contralateral femur. The resection was marginal in order to preserve the knee (Fig. 1). 
3. A marginal resection without reconstruction was undertaken for a metastasis in the acetabulum.

4. An intralesional resection and spinal fusion was undertaken for a metastasis in the lumbar spine (Fig. 2).

The response of the metastases to neoadjuvant chemotherapy was very similar to that of the primary lesions. Two patients had necrosis of more than $90 \%$ in both the primary tumour and the metastases, and the other two had a poor response. Three are alive with no evidence of disease at 5, 7 and 8 years respectively after the surgical treatment. The other died of the disease. Surprisingly, the only death was the case treated by a wide resection; however, this was a chondroblastic osteosarcoma and there was a poor response to chemotherapy in both primary and the metastatic lesions.

\section{DISCUSSION}

To the best of our knowledge, there are no previous reports on this aspect of the treatment for bony metastases of osteosarcoma. There are many reports concerning the incidence and treatment of pulmonary metastases from osteosarcoma, and the role of surgery in managing such patients has been extensively described [11, 12, 17].

Development of bony metastases in patients with osteosarcoma is rare [7, 19]. In our series, only eight of 183 patients with osteosarcoma developed bony metastases. The true incidence may be higher, as bony metastases may develop in the terminal stages of the disease after the development of lung metastases. Bony metastases appeared at a mean of 30 months after diagnosis of the primary lesion. A bone scan is performed routinely in patients with osteosarcoma before starting the treatment protocol in order to detect bony metastases or synchronous polystotic lesions. After finishing the entire treatment protocol 1 year after diagnosis, another bone scan is performed. We believe this second bone scan may not be necessary, as bony metastases - when they occur appear some years later. No bony metastases were detected on routine follow-up bone scans. All bony metastases were diagnosed by clinical symptoms, imaging studies and a biopsy. The first symptom was pain, mainly at night, similar to that with the primary tumour. Special imaging studies should be undertaken when there are symptoms such as unexplained pain, inflammatory pain or neurological symptoms presenting several years after the completion of treatment. Lung metastases rarely may also occur after a 10-year disease-free interval. Half our cases had solitary bony metastases. It is difficult to explain how the tumour cells disseminated in cases of bony metastases without pulmonary involvement, as the lungs should act as a filter in the haematogenous dissemination of the tumour. It is important to confirm the suspected diagnosis of bony metastasis by biopsy. Bone necrosis, traumatic or stress lesions or other benign conditions can simulate bony metastases [3, 4, 10, 15, 18, 20, 21, 22]. Imaging techniques may not always be reliable, and the past history of an osteosarcoma invites the radiologist to over-diagnose possible metastatic lesions. A biopsy was performed in other cases of suspected bony metastasis and the diagnosis was not confirmed.

Treatment of these lesions requires a multidisciplinary approach, and it may be difficult to decide on the most appropriate form of treatment. We have shown, however, that solitary bony metastases can be treated curatively. Three of four cases are alive with no evidence of disease at 5, 7 and 8 years after excision of the metastasis. Response of the 
metastatic lesion to neoadjuvant chemotherapy was similar to that of the primary tumour. Thus, in these cases, we have the advantage of knowing how the tumour will respond to chemotherapy before surgery is undertaken. Survival of this particular subgroup of patients with solitary bony metastases is similar to those in the entire series with osteosarcomas who completed the treatment protocol at our centre - a survival rate of $77 \%$ at 5 years [23].

The benefit of surgery for solitary bony metastases from other tumours, such as renal cell carcinoma, has been described. Baloch et al. [2] found that aggressive surgical management of patients with solitary metastases from renal cell carcinoma can produce good long-term results. Unfortunately, in the cases of multiple bony metastases, treatment could only delay the fatal outcome.

In summary, the appearance of solitary bony metastases during the follow-up of patients with osteosarcoma does not imply a fatal outcome. Such metastases are rare, usually appear late, and we therefore recommend that patients with osteosarcoma should be followed for at least 10 years.

\section{REFERENCES}

1. Bacci G, Ferrari S, Mercuri M, et al (1998) Predictive factors for local recurrence in osteosarcoma. Acta Orthop Scand 69: 230-236

2. Baloch KG, Grimer RJ, Carter SR, Tillman RM (2000) Radical surgery for the solitary bony metastasis from renal-cell carcinoma. J Bone Joint Surg [Br] 82:62-67

3. Ben Ami T, Treves ST, Tumeh S, Cox-Bryan J, McArthy T (1987) Stress fractures after surgery for osteosarcoma: scintigraphic assessment. Radiology 163:157-162

4. Blocklet D, Schoutens A (1999) Massive skeletal metastasis: difficulties of bone scintigraphy, contribution of medullary scintigraphy. Rev Med Brux 20:A165166

5. Cañadell J (1991) Treatment of malignant bone tumors in children and adolescents, 1st edn. EUNSA, Pamplona pp15-76

6. Davies AM, Evans N, Grimer RJ (1988) Fatigue fractures of the proximal tibia simulating malignancy. Br J Radiol 61: 903-908

7. Dangprasert S, Pochanugool L, Likitkitchphysain B (1999) 7 year-survival of 2 cases of multicentric or metastatic osteosarcoma. J Med Assoc Thai 81:728-732

8. Donati D, Di Liddo M, Zavatta M, et al (2000) Massive bone allograft reconstruction in high-grade osteosarcoma. Clin Orthop 377:186-194

9. Girard P, Baldeyrou P, Le Chevalier T, Lemoine G, Tremblay C, Spielmann M, Grunenwald D (1994) Surgical resection of pulmonary metastases. Up to what number? Am J Respir Crit Care Med 149:469-476

10. Grimer RJ, Carter SR, Tillman RM, Spooner D, Mangham DC, Kabukcuoglu Y (1999) Osteosarcoma of the pelvis. J Bone Joint Surg [Br] 81:796-802

11. Kaste CS, Pratt CB, Cain AM, Jones-Wallance DJ, Rao BN (1999) Metastases detected at the time of diagnosis of primary pediatric extremity osteosarcoma at diagnosis. Cancer 86: 1602-1608 
12. Keller SM, Rosenbaum RC, Rosenberg SA (1984) The significance of bone scan abnormalities in patients with primary osteogenic sarcoma. J Surg Oncol 26:122-129

13. Kohler R, Lorge F, Brunat-Mentigny M, Noyer D, Patricot L (1990) Massive bone allografts in children. Int Orthop 14:249-253

14. Lindner N, Ozaki T, Hillman A, Blasius S, Winkelmann W (1996) Adjuvant local treatment of parosteal osteosarcoma. Int Orthop 20:233-236

15. Lowry PA, Carstens MC (1997) Occult trauma mimicking metastases on bone scans in pediatric oncology patients. Pediatr Radiol 27:114-118

16. Natarajan MV, Annamalai K, Williams S, Selvaraj R, Rajagopal TS (2000) Limb salvage in distal tibial osteosarcoma using a custom mega prosthesis. Int Orthop 24:282-284

17. Saetter G, Hoie J, Stenwing AE, Johansson AK, Hannisdal E, Solheim OP (1995) Systemic Relapse of Patients with Osteogenic Sarcoma: Prognostic Factors for Long-term Survival. Cancer 75:1084-1093

18. San-Julian M, Cara JA, Cañadell J (1999) Is amputation still really necessary in any case of osteosarcoma? Rev Med Univ Navarra 43:13-25

19. Shinozaki T, Chigira M, Watanabe H, Kaneko K (1993) Osteosarcoma with multiple skeletal metastases. A case of "nonstochastic" metastasis. Arch Orthop Trauma Surg 112:292-295

20. Snyder CL, Saltzman DA, Ferrell KL, Thompson RC, Leonard AS (1991) A new Approach to the resection of pulmonary osteosarcoma metastases. Results of aggressive metastasectomy. Clin Orthop 270: 247-253

21. Tanaka Y, Ijiri R, Kato K, Nishi T, Nishira H, Aida N (1999) Intrapulmonary lymph nodes in children versus lung metastases. Medical Ped Oncol 33:580-582

22. Tsuchiya H, Tomita K, Mori Y, Asada N, Yamamoto N (1999) Marginal excision for osteosarcoma with caffeine assisted chemotherapy. Clin Orthop 358:27-35

23. Weingrand T, Heyman S, Alavi A (1984) Cold lesions on bone scan in pediatric neoplasms. Clin Nucl Med. 9:125-130 


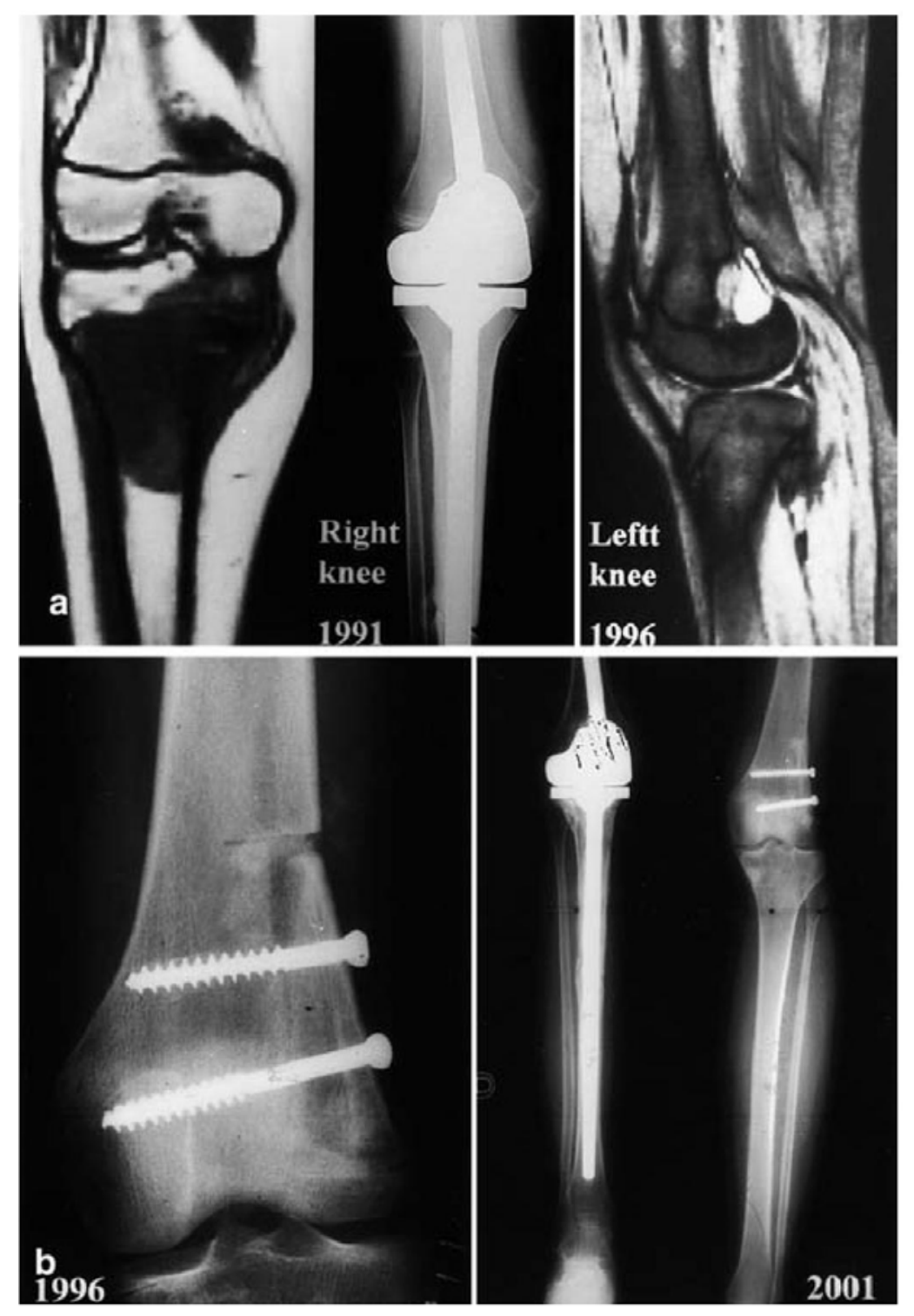

Figure 1 a) A 12-year-old girl affected by osteosarcoma in her right tibia, also involving the epiphysis. She was treated with intraarterial neoadjuvant chemotherapy, surgery and adjuvant chemotherapy. Three years after diagnosis, she developed a pulmonary metastases and a thoracotomy was performed. Six months later, a cerebral metastases was removed. Five years after diagnosis, a bone metastases was detected in the contralateral knee. This lesion did not cross the growth plate. b) After biopsy and intraarterial chemotherapy, the metastasis was removed. Reconstruction was carried out with an intercalary allograft. Five years after surgical procedure for the metastases, there is no evidence of disease. 

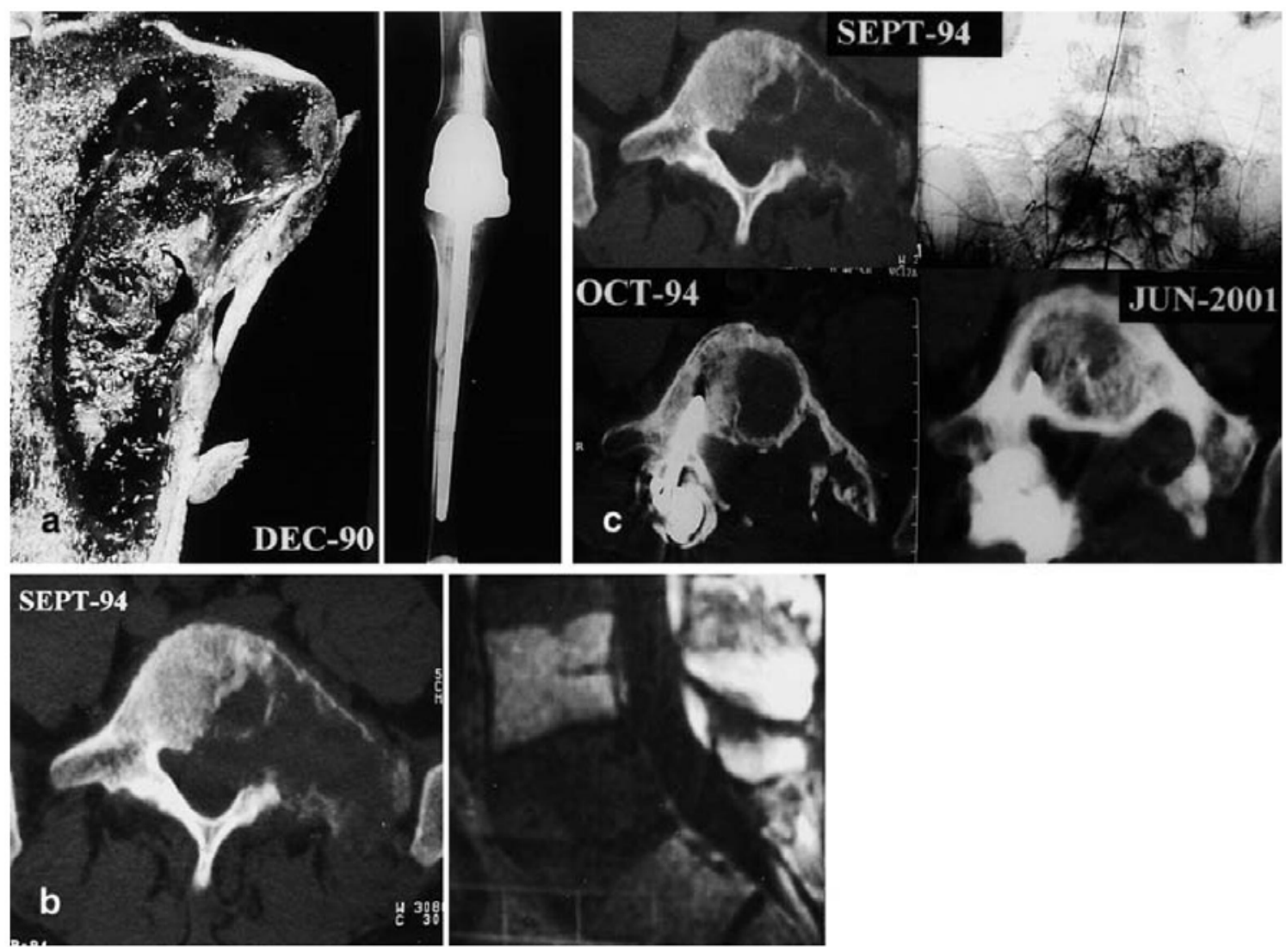

Figure 2 a) This patient was treated in 1990 for a telangiectatic osteosarcoma in the proximal tibia. After intraarterial chemotherapy, the tumour was removed, and the limb was reconstructed with a composite allograft prosthesis. b) Four years later, a bone metastases was detected in the fifth lumbar vertebrae without metastases of the lung. c) After neoadjuvant intraarterial chemotherapy, the metastases was partially excised and a vertebral fusion was carried out. The vertebral tumour showed $100 \%$ necrosis after neoadjuvant chemotherapy. Eight years later, the patient remains free of disease. 